Archives

17| 1996

Hommage à Bernard Lepetit

\title{
Essai d'histoire urbaine. Paris et la Seine
}

Isabelle Backouche

\section{(2) OpenEdition \\ Journals}

\section{Édition électronique}

URL : http://journals.openedition.org/ccrh/2606

DOI : $10.4000 /$ ccrh.2606

ISSN : $1760-7906$

Éditeur

Centre de recherches historiques - EHESS

Édition imprimée

Date de publication : 4 octobre 1996

ISSN : 0990-9141

\section{Référence électronique}

Isabelle Backouche, "Essai d'histoire urbaine. Paris et la Seine ", Les Cahiers du Centre de Recherches Historiques [En ligne], 17| 1996, mis en ligne le 27 février 2009, consulté le 21 avril 2019. URL : http:// journals.openedition.org/ccrh/2606; DOI : 10.4000/ccrh.2606

Ce document a été généré automatiquement le 21 avril 2019

Article L.111-1 du Code de la propriété intellectuelle. 


\title{
Essai d'histoire urbaine. Paris et la Seine
}

\author{
Isabelle Backouche
}

Ce cahier a pour ambition d'exposer la diversité des travaux engagés sous la tutelle de Bernard Lepetit. A mes yeux, il se doit aussi de montrer combien la pratique d'enseignant de Bernard Lepetit appliquée à des recherches doctorales était propice à l'éclosion de nouvelles voies en matière d'histoire. Rien n'était définitivement acquis et sa soif de découvrir de nouvelles façons de faire de l'histoire n'avait d'égale que sa curiosité pour de nouveaux champs.

Il ne se contentait pas de sanctionner un travail et il savait suggérer en donnant le sentiment toujours très stimulant d'apprendre tout autant que nous. Me renvoyant des pages que je lui avais soumises, sa plume ironique notait : « voici mon imprimatur », donnant ainsi un tour particulier à la lecture que je lui demandais en refusant avec dérision une hiérarchie qui à ses yeux perdait de son sens en matière de recherche.

1 Mon idée de départ était de travailler sur la Seine, les études sur le sujet étant rares et les fonds d'archives très riches. Dès notre première rencontre, au printemps 1990, il donnait à ma recherche son ancrage méthodologique : je m'en tiendrai à l'étude du fleuve dans la capitale, et les conséquences de ce choix étaient doubles. La ville devenait mon objet d'étude, la méthode consistait à travailler sur un espace particulier qu'il fallait construire comme objet historique. La fertilité de tels ajustements était certaine et je n'aurai pas la prétention de faire croire qu'elle m'est apparue clairement dès le départ. Au contraire, elle est née du long travail que j'ai mené au côté de Bernard Lepetit, qui savait se laisser convaincre à condition que l'argumentation soit solide.

2 Ainsi, d'emblée, mon travail ne s'inscrivait pas dans le champ de l'histoire urbaine. S'il constitue un "essai d'histoire urbaine", je souhaiterais montrer ici les choix méthodologiques sur lesquels il repose et quelques-uns des résultats obtenus. 


\section{La construction d'un objet historique}

3 Comprendre les relations entre Paris et la Seine en les envisageant dans une dynamique entre le milieu du XVIII ${ }^{e}$ siècle et les années 1850 , tel était mon objectif. Au cœur de ce travail se trouve la tentative de construire un objet historique en conciliant deux exigences: celle de l'analyse historique et celle de la restitution des regards des contemporains.

4 On peut y lire une sorte d'écho permanent entre l'expérience des acteurs et les lignes de force de ma démonstration pour analyser un changement. L'expérience des acteurs apparaît dans toute sa diversité : celle de la population parisienne qui fréquente la Seine et qui s'y rencontre, celle des pouvoirs dans la capitale et, enfin, celle des hommes - qu'ils soient architectes, hommes de lettres ou ingénieurs - et des institutions qui s'intéressent au devenir du fleuve. En m'appuyant sur les pratiques comme sur les représentations de ces hommes, j'ai cherché à réduire au minimum la distance entre les outils de l'analyse et l'expérience des acteurs.

5 Cette attention particulière à la pluralité des relations qu'entretient la ville avec son fleuve m'a permis d'écarter le risque de faire de la Seine le "cadre passif $»^{1}$ de mon étude. Les relations entre l'espace et les hommes forgent des solidarités et participent à la constitution des identités. La forte cohésion des communautés vivant sur les ponts construits en est un exemple. Les relations entre l'espace et le pouvoir légitiment l'autorité du prévôt des marchands face à l'autorité royale toute-puissante dans la capitale. Les relations entre les activités et l'espace fluvial rendent compte de la croissance de la ville, qui ne se résume pas à la délimitation successive de périmètres matérialisés par le rempart ou l'enceinte. Enfin, les relations entre espace et projets urbains mettent en valeur le lien essentiel entre usages et forme fluviale. Comment les premiers ont-ils modelé le fleuve et quels sont les nouveaux critères qui animent les transformations souhaitées, et réalisées, du paysage fluvial?

6 Toutes ces formes de relations jouent entre elles. Parce que la Seine est un espace de travail, les métiers qui y vivent sont directement concernés par toute modification de la forme fluviale. Parce que la Seine est un espace de consommation, les tensions sont nombreuses entre réglementation de l'économie portuaire et aspirations commerciales des marchands. Parce que la Seine est une menace permanente pour les hommes, elle focalise l'attention des pouvoirs et stimule les imaginations pour la vaincre. Ce jeu permanent recouvre ce qu'Augustin Berque appelle « le dialogue architecturé de Paris et de la Seine $»^{2}$, dialogue qui n'est pas figé et dont j'ai souhaité élucider la dynamique.

\section{L'étude d'un espace urbain}

7 On l'aura compris, mon étude repose sur l'analyse d'un espace privilégié. Cette démarche s'est trouvée légitimée par la forte différenciation du fleuve dans l'espace urbain parisien au XVIII ${ }^{\mathrm{e}}$ siècle. Celle-ci s'appuie sur l'originalité des pratiques sociales et économiques que la Seine accueille, sur les rivalités qui s'expriment autour de son contrôle et elle découle de son impact sur la perception de l'espace urbain par les contemporains. L'approche spatiale s'avère en quelque sorte validée par les protagonistes de l'objet historique en cours d'élaboration. Elle a un autre intérêt. 
8 La Seine est un espace urbain qui a deux qualités du point de vue de l'historien. C'est un espace circonscrit dans sa topographie mais aussi par les pratiques qu'il abrite et par les représentations qui s'y attachent. Cette conjonction le donne à voir comme un véritable "précipité » de la ville d'Ancien Régime. La question cruciale de l'approvisionnement à l'échelle d'une ville comme Paris s'y rencontre de façon lancinante, le fractionnement des compétences se double d'une réglementation rigide pour faire face aux aspirations contradictoires des différentes activités exercées sur le fleuve, la précarité de la vie humaine confrontée aux dangers du fleuve rappelle l'instabilité qui caractérise l'arrivée de migrants toujours plus nombreux. Mais la Seine est aussi un espace urbain ouvert et très attractif, un lieu de rencontre de la société parisienne qui s'y retrouve pour consommer, travailler et s'amuser. Sa traversée dans la capitale donne naissance à des paysages contrastés et sa présence dans la ville organise la perception de l'espace urbain, dans la vie quotidienne comme dans les ouvrages littéraires ou les projets des hommes de l'art.

9 Cette double qualité permet de rompre avec les deux pôles traditionnellement privilégiés pour cerner les phénomènes urbains. Ni le quartier, réalité mouvante et trop souvent surimposée sur l'objet urbain, ni la ville, entité globale relativement abstraite, ne forment le cadre de mon étude. Cette particularité du fleuve permet de concilier deux échelles d'analyse : la ville et le fleuve, d'une part; le Parisien et le fleuve, d'autre part. Ces deux types de relations sont constitutifs du fait urbain et ils rendent comptent de sa complexité et de sa richesse. Le fleuve comme la ville ne sont pas seulement les limites commodes d'un champ d'observation. Ce sont des territoires imbriqués, en perpétuelle composition du fait de la diversité des enjeux dont ils sont l'objet, enjeux qui ne sont perceptibles qu'au travers d'une lecture concomitante de réalités trop souvent isolées pour les nécessités de l'analyse.

\section{En guise de résultats}

10 Quelques résultats illustreront mon argumentation. La Seine est tout d'abord un espace partagé. Par tous les Parisiens, parce qu'elle leur apporte leur subsistance et l'eau qu'ils boivent. Par de nombreux métiers qui s'y côtoient et ne peuvent se passer du site fluvial pour des raisons techniques. Par le prévôt des marchands et le lieutenant général de police qui, sans cesse, défendent leurs compétences que, justement, l'espace différencie. Le fleuve est enfin partagé physiquement en « bassins » délimités par les ponts.

11 Dans chacun de ces cas, la dépendance vis-à-vis du fleuve prend une signification différente et c'est précisément cette multiplicité de sens qui fabrique la ville et forge l'identité urbaine. A l'égard de la collectivité, il s'agit d'assurer la paix sociale et de ne pas réveiller la peur de la disette. Envers les Parisiens qui y travaillent, elle cimente des dynasties fluviales et explique leur ardeur à vouloir rester sur les rives du fleuve. Pour les pouvoirs, cette dépendance recoupe la notion de légitimité et elle entraîne une forte résistance à toute innovation qui enlèverait au fleuve sa suprématie, notamment en matière de distribution de l'eau.

12 Ce partage de l'espace fluvial, et la dépendance qui en découle, prennent un sens nouveau à partir du milieu du XvIII ${ }^{e}$ siècle. L'idée d'une rivalité entre les usages se fait plus nette et, ici encore, elle recouvre plusieurs réalités. Des tensions de plus en plus fortes s'exercent pour occuper l'espace fluvial et de nombreux conflits opposent 
systématiquement les usages sédentaires - moulins, pêcheurs, bateaux à lessives, bateaux de bains - à la navigation. La cohabitation des différents types de commerce sur les bords de la Seine - depuis l'échoppe jusqu'au magasin de luxe - donne lieu aussi à des rivalités qui s'expriment par l'incompatibilité entre les clientèles et les nuisances provoquées par l'affluence. Autre affrontement, celui des marchands des ports, qui supportent de plus en plus mal la rigidité des règlements qui imposent un contingentement de leurs ventes justifié par le manque d'espace pour donner libre cours à une concurrence qu'ils appellent de tous leurs vœux.

13 Ces conflits autour de l'espace fluvial aboutissent à la remise en cause de son organisation dès le milieu du XVIII ${ }^{e}$ siècle. Cette idée n'est pas seulement le fruit de l'analyse historique, mais elle est perceptible à divers titres dans les évolutions qui marquent l'attitude des pouvoirs, les idées exprimées dans les projets et la perception de l'espace fluvial. Les arbitrages autour des conflits aboutissent à une hiérarchisation des usages au profit de la navigation. Les réticences sont de plus en plus fortes à tolérer de nouveaux établissements sédentaires, qui doivent à la fois satisfaire des besoins essentiels comme la fourniture de l'eau ou la multiplication des équipements en bains, et l'exigence de fluidité le long de l'espace fluvial promu avant tout comme un lieu de circulation. Dès le milieu du siècle, une spécialisation à l'échelle du fleuve s'esquisse. Des activités cousines sont regroupées comme la vente du bois et le déchirage des bateaux dans l'île des Cygnes, alors que d'autres sont exclues du milieu urbain au nom de considérations sanitaires. Cette réorganisation des pratiques, synonyme de rejet des activités encombrantes ou polluantes, entraîne une dilatation de l'espace fluvial utile à la ville et montre le lien étroit qui existe entre croissance de la ville et distribution des pratiques.

Enfin, cette remise en cause se manifeste aussi par la prolifération de projets qui associent étroitement les considérations esthétiques auxquelles la forme du fleuve doit répondre - ouverture de la ville sur l'eau, création de perspectives - et les nécessités d'une meilleure organisation de l'espace fluvial au nom de critères tels que la salubrité, la circulation, la lutte contre les crues. A la diversité des auteurs qui se penchent sur la question et à la nouveauté des arguments qui animent les propositions, il faut encore ajouter l'exigence sans cesse réaffirmée de travailler à l'échelle de tout le fleuve. Apparaît alors une globalisation de l'aménagement urbain, qui donne naissance en 1769 au premier plan d'aménagement à l'échelle de toute la ville centré sur le fleuve.

\section{La mise en mouvement d'un espace urbain}

Le partage, la confrontation et la remise en cause de cet espace urbain se conjuguent pour impulser sa mise en mouvement. Elle prend deux formes principales. L'économie fluviale, tout d'abord, se transforme. Les nombreux métiers installés sur le fleuve et la vente des consommations dans les ports cèdent la place à une économie de flux qui privilégie l'arrivée des marchandises, l'exportation des produits fabriqués et la circulation des voyageurs dans la ville et au-delà.

La forme fluviale se modifie également et s'organise peu à peu pour satisfaire les exigences de la circulation fluviale et urbaine. Elle n'est plus le produit d'une construction qui impliquait la diversité des acteurs, diversité qui avait donné naissance à toute une gamme de paysages. Le Pont-Neuf construit par le roi, l'Hôtel de Ville où siège le prévôt des marchands, le lotissement de l'île Saint-Louis ou la construction des maisons 
$\mathrm{du}$ pont au Change par leurs habitants étaient autant de traces du foisonnement d'intérêts que la Seine accueillait. Ainsi, évolution de la forme fluviale et réorganisation de l'économie sont intimement liées, donnant un sens nouveau aux relations entre la ville et le fleuve.

Replacées dans le temps et rapportées aux processus qui les commandent, les formes urbaines méritent l'attention de l'historien pour comprendre l'évolution d'une société aux prises avec son espace. Trois faits majeurs caractérisent le cas que j'ai étudié et convergent vers l'idée d'une rupture entre les Parisiens et la Seine. Un changement d'échelle du rôle du fleuve qui s'affirme comme un moyen de transport à vocation nationale, une perte de l'identité urbaine de la Seine soumise à des autorités administratives étrangères à la ville et, enfin, une unité physique qui l'emporte sur les pratiques pour différencier cet espace dans Paris.

Trois types de mécanismes qui s'inscrivent dans des échelles contextuelles emboitées sont à l'origine de cette rupture.

Les conflits repérés sur l'espace fluvial et les propositions émises au sujet de son rôle dans la ville autour des années 1750 peuvent s'interpréter comme une sorte d'appel au changement. Les contraintes spatiales sur le fleuve comme sur les ports, les accrochages entre les acteurs politiques, les velléités des marchands face à la réglementation qui bride leur activité, la condamnation de l'entassement des hommes et des activités signalent une telle aspiration. Elle s'enracine dans les divers aspects de la vie sur le fleuve et a déterminé le point de départ de mon étude.

Ce désir de changement s'exacerbe au milieu du siècle en raison de la pression démographique que connaît la capitale. Il se heurte à l'inertie des techniques, qu'elles concernent la navigation ou la distribution de l'eau, et à la sédimentation de textes juridiques qui brouillent la répartition des compétences sur le fleuve. Il est par contre renforcé par des initiatives telles que les édits de Turgot de 1776 qui consacrent la liberté de circulation des marchandises ou par le succès de la construction d'une nouvelle place royale dédiée à Louis XV. Tous ces facteurs déplacent le point de vue de l'analyse et font appel à un contexte parisien plus large mais tout aussi essentiel.

21 Enfin, les transformations nées de la Révolution et de l'Empire, trop souvent considérées comme des points de rupture entre un « avant » et un « après » qui n'auraient aucun lien entre eux, doivent être lues comme des accélérateurs de changements en gestation.

La disparition de la représentation municipale parisienne qui s'identifiait avec l'espace fluvial va de pair avec l'élargissement du cadre de contrôle du fleuve à l'échelle du département, la fragmentation des compétences entre les prérogatives du préfet de police sur les pratiques et la main-mise des ingénieurs des ponts et chaussées sur les aménagements fluviaux, l'insertion de la Seine dans un réseau de voies fluviales artificielles que forment les canaux, le dynamisme des milieux d'affaires qui financent la construction des ponts et des ports, et, enfin, la rivalité induite par l'avènement du chemin de fer, tous ces facteurs forment le troisième faisceau de causes sur lesquelles s'appuie ma démonstration. 


\section{L'autonomie des processus urbains}

23

Cette complexité du processus qui affecte les relations entre Paris et son fleuve désigne l'interdépendance entre plusieurs niveaux de la réalité. Fédérés sous une problématique propre à l'objet urbain, ils légitiment la spécificité de l'analyse urbaine.

Il ne s'agit pas seulement de comprendre le poids économique du fleuve, ou d'étudier les modalités de son contrôle, ou encore de saisir la diversité sociale qu'il accueille. Ce sont surtout les interférences entre ces domaines, trop souvent isolés par une historiographie empreinte de catégories utiles à l'analyse, qui ont retenu mon attention. Un tel cloisonnement masque en effet la complexité urbaine et empêche de saisir des processus figés à l'avance par la mise en œuvre de l'étude. Se placer à deux moments donnés, dans le cas de mon étude les années 1750 et le milieu du xixe siècle, et comparer, à tour de rôle, des données économiques, des groupes sociaux, des moyens de régulation, des formes urbaines ou encore des projets d'aménagement ne pouvait satisfaire mon objectif. Tout mon effort a consisté à analyser la dynamique entre ces différents niveaux de la réalité qui obéissent à des temporalités distinctes et font vivre des représentations souvent opposées.

Les conflits en sont une trace que l'historien peut appréhender. Conflits d'usages entre les acteurs sociaux, conflits entre les agents de l'économie urbaine tels que le prévôt, le marchand ou le consommateur, conflits de pouvoir entre les différentes autorités qui ont un droit de regard sur le fleuve, conflits intellectuels dans l'affrontement sur les projets et leur réalisation. Le conflit a un autre intérêt. Résultant de la confrontation entre plusieurs réalités et des décalages entre les représentations des acteurs, il oriente l'attention de l'historien sur des questions qui ne sont pas étrangères à la société observée. Ainsi, il rapproche le questionnement de l'analyse historique des préoccupations des contemporains, ces hommes que Bernard Lepetit nommait volontiers les « indigènes ».

Par ailleurs, cette étude impose de revoir les coupures chronologiques traditionnelles afin de satisfaire les interrogations qui s'attachent à la ville et à son devenir. La pluralité des facteurs invoqués pour rendre compte de la dynamique de l'espace fluvial rappelle la profondeur de l'inscription temporelle des transformations urbaines et leur rythme saccadé.

Commentant les repères temporels que L.-S. Mercier utilise dans son Tableau de Paris, J.-C. Perrot introduit une telle idée :

« Naguère, répète-t-il sans cesse, ou bien encore il y a trente ans. S'il fallait accorder quelque crédit à ce laps de temps, alors les années 1750-1760 constitueraient bien la charnière de deux époques indûment confondues sous l'étiquette séculaire. C'est aux historiens de reprendre ce problème de chronologie $»^{3}$.

Le processus mis en évidence sur la Seine est en quelque sorte une réponse à l'appel de J.C. Perrot. En effet, c'est dans les années 1750-1760 que se cristallisent les détonateurs d'une dynamique propre à l'espace fluvial. De même, le poids des transformations hausmanniennes marque profondément les études sur la capitale. Les années 1860 sont le plus souvent considérées comme le point de départ d'une remise en cause systématique $\mathrm{du}$ vieux Paris. En fait, au milieu du xIx ${ }^{\mathrm{e}}$ siècle, la Seine a largement entamé une telle évolution, et le Second Empire ne fera que retoucher un paysage déjà entièrement refondu. 
Ainsi, le cas de l'espace fluvial oblige à repenser la chronologie des transformations de la capitale. Il dévoile aussi leur rapidité. A ce titre, les projets des années 1840 qui souhaitent redonner au fleuve son pouvoir d'attraction sur la société et l'économie parisiennes permettent de mesurer l'ampleur de la dynamique ainsi que la conscience aiguë qu'ont les contemporains de ses effets. Ces projets émanant d'architectes et d'hommes politiques en constituent les seules traces. Si, au XVIII ${ }^{e}$ siècle, les conflits permettaient de saisir le point de vue de tous les acteurs, au contraire, le dépassement des conflits, au prix de la disparition de bien des usagers, rend beaucoup plus difficile la confrontation des représentations et des aspirations au siècle suivant. L'historien travaille sur un matériau qui est le produit de l'activité humaine et parfois certaines questions trouvent difficilement leur réponse. La logique qui prévaut sur le fleuve au milieu du XIX ${ }^{e}$ siècle est celle des ingénieurs des ponts et chaussées et du pouvoir préfectoral. Etrangère aux aspirations des Parisiens, elle ne doit pas laisser supposer qu'elle est seule à exister et on aurait aimer pouvoir cerner de plus près les réactions, et la nouvelle «conscience du fleuve »-si elle existe - de ceux dont la voix a disparu des bords du fleuve. Cette interrogation ouvre sur une autre histoire, qui n'entrait pas dans mon projet, celle de l'évolution des perceptions d'un espace urbain sur le long terme.

\section{NOTES}

1. Louis Bergeron et Marcel Roncayolo regrettaient en 1974 cette tendance : "L'étude des villes est trop souvent le «sous-produit " ou le cadre "passif » de recherches qui obéissent à des préoccupations thématiques différentes ", «De la ville pré-industrielle à la ville industrielle. Essai sur l'historiographie française ", Quaderni Storici, 27, 1974, p. 828.

2. A. Berque, Du geste à la cité. Formes urbaines et lien social au Japon, Paris, Gallimard, 1993, p. 69.

3. J.-C. Perrot, « Rapports sociaux et villes au XVIII ${ }^{\mathrm{e}}$ siècle », Annales ESC, mars-avril 1968, p. 250.

\section{AUTEUR}

\section{ISABELLE BACKOUCHE}

Agrégée d'histoire.

EHESS, sous la direction de Bernard Lepetit :

Thèse soutenue, 2 décembre 1995, La Seine et Paris, 1750-1850. Pratiques, aménagements, représentations.

Maître de conférences, histoire contemporaine, université de Paris XIII, 1996. 